\title{
Strain in Shear, and Material Behaviour in Incremental Forming
}

\author{
W. C. Emmens ${ }^{1, a}$, A. H. van den Boogaard ${ }^{2, b}$ \\ ${ }^{1}$ CORUS RD\&T; P.O.Box 10.000, 1970 CA IJmuiden, the Netherlands \\ ${ }^{2}$ University of Twente; P.O.Box 217, 7500 AE Enschede, the Netherlands \\ awilko.emmens@corusgroup.com, ${ }^{b}$ A.H.vandenBoogaard@utwente.nl
}

Keywords: Shear Forming, Incremental Forming, Material Behaviour

\begin{abstract}
This paper discusses some consequences of forming by shear, a situation that is sometimes claimed to occur in incremental forming. The determination of the principal strains and principal directions is discussed in detail. Two methods are presented: using a circular grid (although simulated on the computer), and by deriving formulae from the theory; both yield identical results. The strains assuming forming by shear are found to be (much) higher than in situations of forming by stretch. This affects notably more fundamental studies on material behaviour in incremental forming. The effects are illustrated using experimental data obtained with pre-stressed material.
\end{abstract}

\section{Introduction and definition of the problem}

Incremental forming is the name of a variety of forming processes characterized by the fact that the product is not formed as a whole, but that at any time only a small part is actually being deformed. Of special interest is so-called incremental sheet forming (ISF) of which several varieties exist, that are carried out by moving a steel punch or roller by a CNC-machine over a metal sheet (see [1] for an overview).

Many authors claim that in incremental forming deformation takes place by shear instead of stretching (see for example [2]), despite the fact that there is no direct experimental evidence for that. This seems to be led by drawing a parallel with shear-spinning, which indeed has many features in common. If this is indeed the case however then there are some consequences that seem to be overlooked. This concerns the principal directions of deformation in the material.

The principal directions in forming are an orthogonal set of directions in the material that remain orthogonal in the forming operation (such a set always exists, at least locally). The strains in these principal directions are called principal strains. This may seem academically to many readers but it is of importance when one wants to carry out a more fundamental analysis of the process, specially if this is related to material behaviour.

Material models for work hardening are commonly related to an equivalent (or effective) plastic strain. E.g. for the von Mises yield function, the equivalent strain rate is defined as $\dot{\varepsilon}_{\text {eff }}=$ $\sqrt{ }\left(2 / 3 . \dot{\varepsilon}_{\mathrm{ij}} \dot{\varepsilon}_{\mathrm{ij}}\right)$. For proportional deformation the equivalent (von Mises) strain can easily be expressed in the principal directions as $\varepsilon_{\text {eff }}=\sqrt{ }\left(2 / 3\left(\varepsilon_{1}{ }^{2}+\varepsilon_{2}{ }^{2}+\varepsilon_{3}{ }^{2}\right)\right)$. In experimental determination of strains in sheet metal forming often the 'principal' strains on the surface are determined from an etched circular grid. However if deformation is dominated by out-of-plane shear (as claimed for incremental sheet forming), the real principal strains are not in the plane of the surface and the 'principal' strains determined in this manner are incorrect. A last point to be mentioned is that for large deformations the definition of strain is not unique. For large plastic deformations it is common to use the logarithmic strain, such that constant volume deformation can be described by $\varepsilon_{1}+\varepsilon_{2}+\varepsilon_{3}=0$. However this is only valid for the correct principal strains.

The fact that there seems to be a confusion about the determination of the principal strains is by itself understandable. The reader should keep in mind that the forming technology in general as has been developed in the previous century is purely based on forming by stretching, and for example the concept of FLC is based on that. That has influenced our way of thinking and how we intuitively interpret results and numerical data. 
This paper now will first discuss the determination of the principal directions and principal strains in case of forming by shear. Secondly, the relation with the equivalent strain will be presented.

\section{Basics: forming by shear}

Fig. 1 shows the difference between forming by stretching and forming by shear. In forming by stretching (left) the original cross sections of the sheet material remain perpendicular to the surface, while in forming by shear (right) the original cross-sections keep their original orientation. So in forming by stretching the original cross-sections remain perpendicular to the surface, and it is easy to see the link with principal directions: one of the principal directions is perpendicular to the surface while the other two are in the plane of the sheet. In forming by shear the original cross-sections change their orientation relative to the surface and do not represent one of the principal directions.
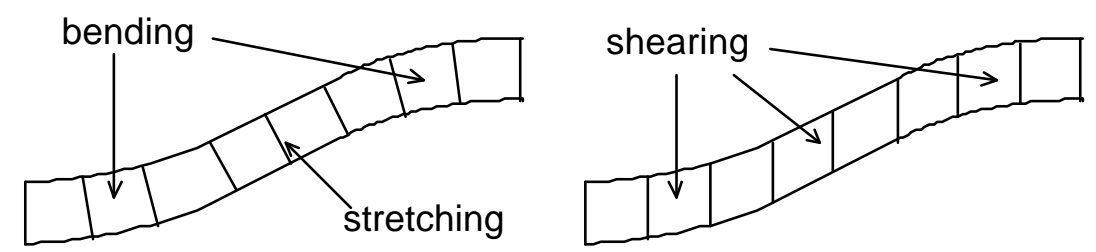

Figure 1. Difference between forming by stretch (left) and forming by shear (right) illustrated with a S-shaped specimen. Note the difference in orientation of the original vertical cross-sections.
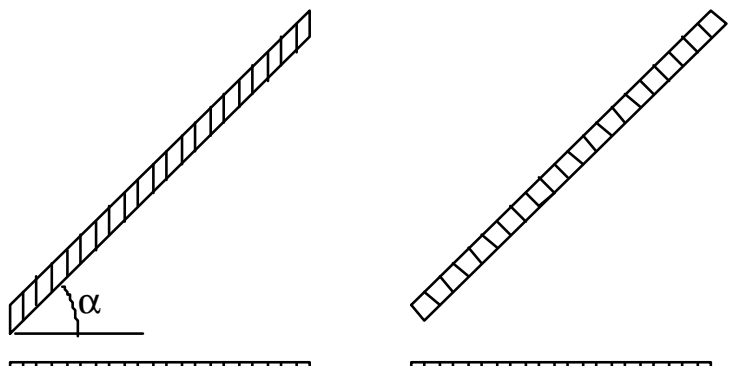

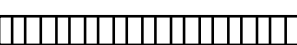

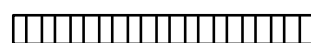

Figure 2. A flat piece of material has become part of an angled wall. Left: proposed situation in incremental forming: forming by shear. Right: similar situation assuming forming by stretch. Note the difference in edges of the tilted part. The hatches illustrate what happens to the original crosssections. This figure also defines the technological shear angle $\alpha$.

Note that in case of forming by stretch (Fig. 2, right) the major strain can simply be derived from the change in macroscopic length (which can be derived from the well-known sine formula):

$$
\varepsilon_{\text {major }}=\ln (1 / \cos (\alpha))
$$

and the direction of the major strain is parallel to the sheet surface. This situation of forming by stretch will be referred to as method A throughout this paper (as for example in figure 5: lines A1 and A2) and is supposed to be well-known by the reader.

Two methods related to forming by shear (labelled B and C) will be presented following; these will be presented more general as an illustration how such problems can be handled so that the readers can use them in other situations as well. 


\section{Method B: forming by shear, single-step method}

The single step method is a simple method, in general easy to use, that only considers the initial and final configuration and is therefore strongly related to the experimental determination of strains based on images of the undeformed and deformed work piece (e.g. using grids). This method however has its limitations, therefore another method (labelled C) will be presented below as well.

Circular grid. The simplest method of obtaining the principal directions and principal strains is using a circular grid method. A sufficiently small circle on the deforming material will be changed into an ellipse, and the two axes of that ellipse represent the two principal strains and principal directions. The third principal direction is assumed to be oriented perpendicular to the plane of the circle, and the third principal strain can be obtained by assuming constant volume.

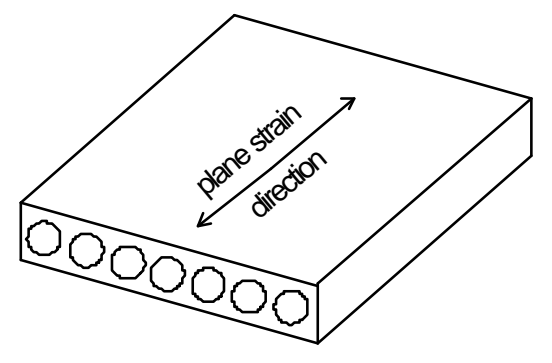

Figure 3. To use the grid method correctly a grid is proposed to be applied on a cross-section of the sheet.

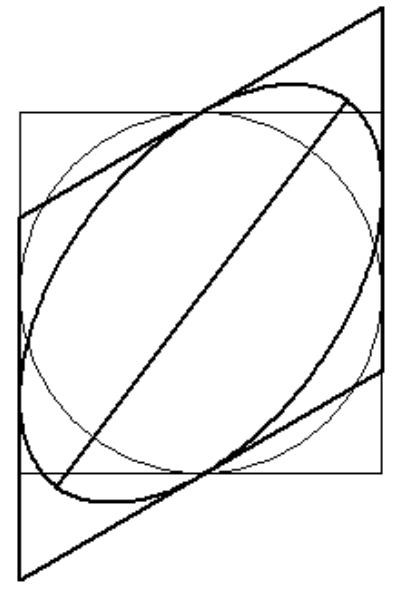

Figure 4. Example of a computer simulation. The original square and circle are in thin lines. The sheared square (now a parallelogram) and circle (now an ellipse, note the long axis) are in thick lines; the shear angle $\alpha$ (as defined in figure 2) is $30^{\circ}$.

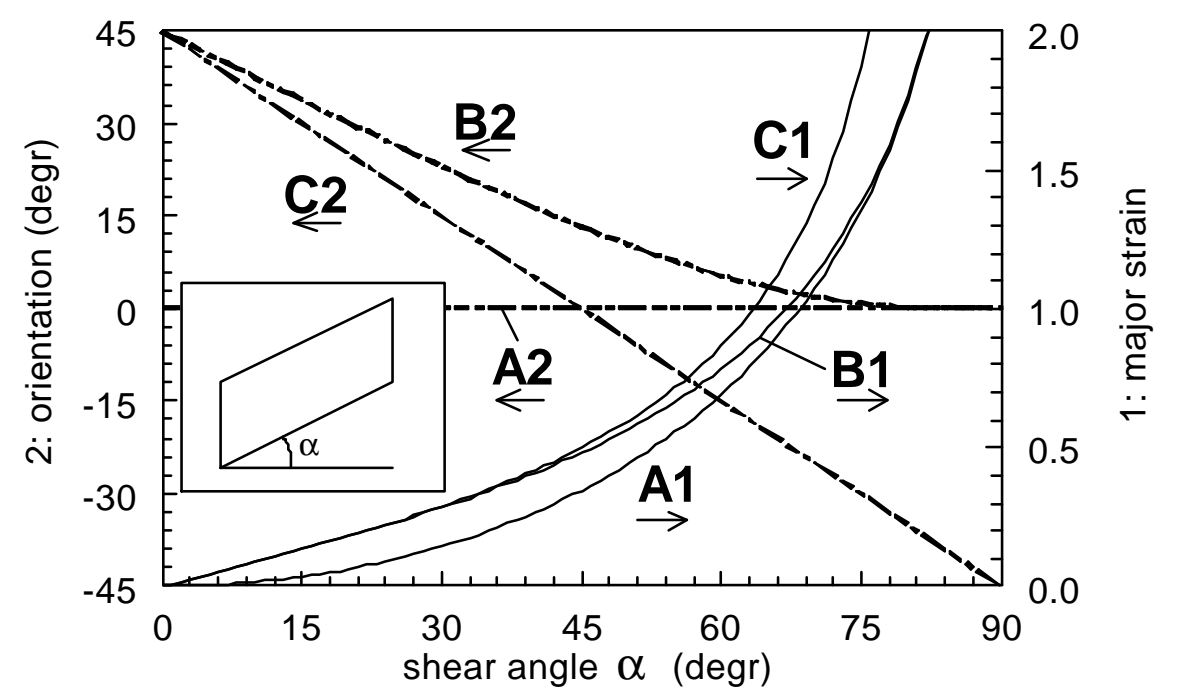

Figure 5. Results as a function of the technical shear angle. The six lines present:

A1: major strain assuming stretch according to Eq. 1 .

B1: major strain assuming shear, single step method.

C1: cumulative 'major' strain, incremental method.

A2: principal direction relative to sheet surface assuming stretch.

B2: principal direction of total strain relative to sheet surface, shear, single step method.

C2: principal direction of shear increments (rate) relative to sheet surface, see text. 
The problem now is that we have to place circles on a cross-section as shown in Fig. 3 which is not particularly easy to accomplish. So the process is simulated on the computer. This can be done because we assume to know the way the sheet is deforming (by shear).

A computer program has been written that shears a square. A circle is sheared in the same way and the long axis of the resulting ellipse is determined. This is done by determining the location on the ellipse that has the longest distance to the origin (with a resolution of $0.01^{\circ}$ ). The orientation of the long axis is the principal direction, and the le ngth gives the corresponding strain (in this case the major strain). Fig. 4 shows the resulting plot.

The results are presented in Fig. 5. Line A1 shows the major strain as in stretch (Eq. (1)), line B1 the major strain as obtained with the computer simulation. There is a clear difference between these two. The strain in shear is always higher and starts as a linear function of the shear angle, while the strain in stretch starts as a parabolic function of the shear angle. For higher shear angles the difference becomes smaller. This is easy to understand from line B2. This shows that for high shear angles the principal direction becomes more or less parallel to the surface so that the forming operation resembles simple stretching (lines $\mathrm{C} 1$ and $\mathrm{C} 2$ will be dis cussed later).

Theoretical method: basics. The theoretical method presented here is based on 2D-analysis, which is only valid if at least one of the principal directions is known. The other two lie in a plane perpendicular to that direction. If none of the principal directions is known beforehand a full 3D analysis is required. The reader is expected to be familiar with fundamental mechanics and the tensor representation of strains; these can be found in good text books and university courses e.g. [3]. This section will present general formulae, the next section will apply these to our problem.
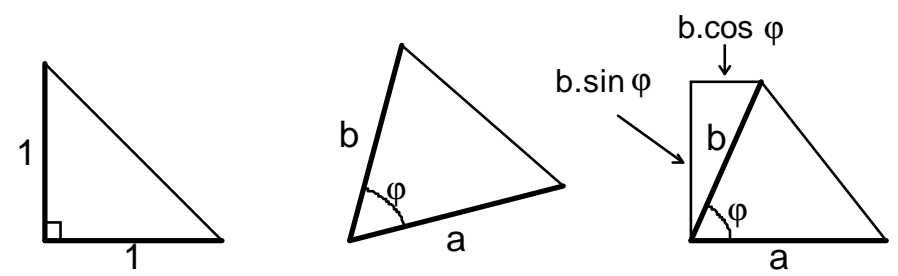

Figure 6. Some definitions used in the text. An original rectangular triangle (left) is deformed into an oblique triangle (centre), a coordinate system is placed along one of the sides (right).

The method is more or less based on the practical method of using a rectangular grid. Consider an original rectangular triangle (for example a part of a rectangular grid) as shown in Fig. 6, left. For convenience we assume the lengths of the rectangular sides to be 1 . After deformation this triangle is deformed in general into a triangle as shown in the Fig. 6, centre, with sides $a$ and $b$ and enclosed angle $\varphi$. For further analysis we assume an orthogonal coordinate system with one axis parallel to side a (very arbitrarily!) as shown in the Fig. 6, right. Note that $\mathrm{a}$ and $\mathrm{b}$ are in fact so-called stretch ratios defined as:

$$
\text { stretch ratio }=\frac{\text { new length }}{\text { original length }}
$$

The principal strains can now be obtained by deriving the eigenvalues of either the left or right Cauchy-Green deformation tensor and this yields:

$$
\varepsilon_{1,2}=\ln \left(\sqrt{\lambda_{1,2}}\right)=\ln \left(\lambda_{1,2}\right) / 2 \quad \lambda_{1,2}=\frac{\left(a^{2}+b^{2}\right) \pm \sqrt{\left(a^{2}+b^{2}\right)^{2}-4 \cdot(\text { a.b. } \sin (\varphi))^{2}}}{2}
$$

The principal strain directions can be obtained by deriving the eigenvectors of the left CauchyGreen deformation tensor and this yields ( $t$ is the tangent of the principal direction relative to side a):

$$
\mathrm{t}_{1,2}=\frac{-\mathrm{Q} \pm \sqrt{\mathrm{Q}^{2}+4}}{2} \quad \mathrm{Q}=\frac{\mathrm{a}^{2}+\mathrm{b}^{2} \cdot\left(\cos (\varphi)^{2}-\sin (\varphi)^{2}\right)}{\mathrm{b}^{2} \cdot \sin (\varphi) \cdot \cos (\varphi)}=2 \cdot \frac{\mathrm{a}^{2}+\mathrm{b}^{2} \cdot \cos (2 \varphi)}{\mathrm{b}^{2} \cdot \sin (2 \varphi)}
$$


Theoretical method: application. We now use the former to derive the principal directions and principal strains to our situation of forming by shear. For reasons of convenience and compatibility we will take the surface of the sheet as the reference plane (represented by the vector ' $a$ ' in Fig.6, right). Using the definitions from Fig. 6 we get specifically (see also Fig. 7):

$$
\mathrm{b}=1 ; \quad \varphi=\pi / 2-\alpha ; \quad \mathrm{a}=1 / \cos (\alpha)=1 / \sin (\varphi) ;
$$

in which $\alpha$ is the 'technical' shear angle as defined in Fig. 2.

Using these definitions we finally get the same results (numerically) as already obtained by the grid method and presented in Fig. 5; for example Eq. 2 thus becomes:

$$
\varepsilon_{1,2}=\ln \left(\lambda_{1,2}\right) / 2 \quad \lambda_{1,2}=\frac{\mathrm{Z} \pm \sqrt{\mathrm{Z}^{2}-4}}{2} \quad \mathrm{Z}=1+\frac{1}{(\cos (\alpha))^{2}}
$$

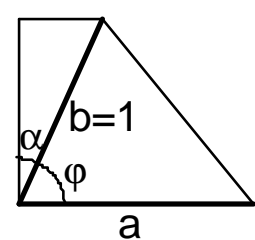

Figure 7. Situation for forming by shear as proposed for incremental forming; compare to Fig. 6.

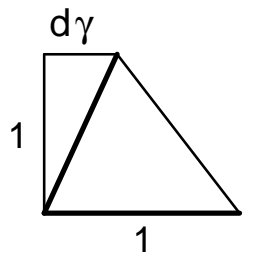

Figure 8. More fundamental presentation of shear used for the incremental theoretical pproach.

\section{Method C: forming by shear, incremental method}

The calculations presented above have a fundamental limitation: they relate the undeformed situation to the final deformed situation directly. This however is only valid in situations of straight strain-path, meaning that the principal directions are fixed to the material, and that the ratios $\varepsilon_{1}: \varepsilon_{2}$ : $\varepsilon_{3}$ remain constant during the forming operation. This is not the case here: Fig. 5 shows that the principal directions rotate. This implies that the strain history (determining the level of work hardening) of the material is more severe than expected from the single-step method, and we have to determine the total equivalent strain by integrating small increments. For reasons of compatibility with the former results this will be treated following as a 'cumulative major' strain; this has no physical meaning but is allowed here as in this particular case it is proportional to the equivalent strain.

Circular grid. The problem has been tackled by performing the simulation as shown in Fig. 4 for small increments. This means that the shear process is carried out in small increments of $0.1^{\circ}$ only, in each step a new circle is plotted, the (slightly) deformed circle after the small shear increment is determined, and the major strain and the principal direction are measured. Finally the strain increments are added to obtain the cumulative major strain.

The results are shown in Fig. 5, line $\mathrm{C} 1$. One sees clearly that for larger shear angles $\left(\mathrm{say}>60^{\circ}\right.$ ) the difference between lines C1 (incremental) and B1 (single step) is significant.

Noteworthy is that the principal direction remains at an angle of $45^{\circ}$ relative to side $\mathrm{b}$ all the time (note: side $b$ is NOT the sheet surface!), which means that in Fig. 4 the principal directions do not rotate at all! This seems in contradiction to the single step method. However careful examination of the results show that there is a slight rotation of the principal direction, to be precise $1 / 4^{\circ}$ for each degree of rotation of the shear angle. For not-too-large shear angles the rotation in the single-step method relative to side $b$ equals the sum of all incremental rotations relative to side $b$.

Line C2 in Fig. 5 shows the principal direction relative to side a being the sheet surface. That di- 
rection seems to rotate, but in fact line $\mathrm{C} 2$ only reflects the rotation of side a relative to side $\mathrm{b}$ due to the shear process (see Fig. 7 for the definitions of sides $a$ and $b$ ).

Theoretical approach. For the theoretical approach of this situation we use a slightly different and more fundamental presentation of shear as shown in Fig. 8. Note that in fact compared to Fig. 7 the sides $a$ and $b$ have been swapped. Note also that in this situation the shear parameter $d y$ is in fact a length, not an angle. As we study small increments only we may assume $\mathrm{d} \gamma<<1$. This finally yields:

$$
\mathrm{d} \varepsilon_{\text {major }}=\mathrm{d} \gamma / 2
$$

The total cumulative 'major strain' can now be obtained by integrating Eq. 6 over the total shear distance being equal to $\tan (\alpha)$; this yields:

$$
\varepsilon_{\text {major,cumulative }}=\tan (\alpha) / 2
$$

This relation yields exactly the same numerical results as found by the circular grid method which have been presented in Fig. 5 by line C1.

For proportional deformation lines B1 and C1 would coincide. The difference between both lines can be used as a measure for the non-proportionality as suggested by one of the authors in [4].

\section{Illustration using experimental data}

The effect of the relations above will now be illustrated using data of pre-stressed material. As at the time of writing the authors have not been able to carry out relevant experiments themselves we will use data found in the literature. In 2003 Hagan and Jeswiet [5] have carried out tensile tests on pre-stressed material. They made products with hrge flat sides with variable slopes by incremental forming and took tensile test samples from those sides. The original tensile curves have been published in [5] and are reprocessed here. The results are used to create combined curves by offsetting the original tensile test curves by the level of pre-strain as proposed in [5]. Note however that in this report the von-Mises strain is used as an offset, and not the major strain as used by Hagan \& Jeswiet. This means that the results presented here differ slightly from those in [5].

The results are presented in Fig. 9. The top graph (A) shows the tensile test data using the strains from curve A1 in Fig. 9 as an offset (just as Hagan \& Jeswiet have done) assuming forming by stretch. The centre graph (B) shows the data using the strains from curve B1 as an offset, assuming forming by shear and using the single-step method. Note the difference, notably at smaller shear angles. The lower graph (C) finally uses the incremental shear strains from curve $\mathrm{C} 1$ as an offset. The difference with graph $\mathrm{B}$ is mainly restricted to larger shear angles.

A Hollomon (Ludwik-Nadai) fit $\left(\sigma=K . \varepsilon^{n}\right)$ has been obtained for all three situations, these are shown in Fig. 9 as well. This follows Hagan \& Jeswiet, although it is not known if the particular material is supposed to obey the Hollomon equation for large strains. The parameters found are also presented in Table 1.

Table 1. Parameters of a Hollomon fit (Ludwik-Nadai) through the data presented in Fig. 9

\begin{tabular}{|l|c|c|}
\hline \multicolumn{1}{|c|}{ Method } & $\mathrm{K}[\mathrm{MPa}]$ & $\mathrm{n}$ \\
\hline A: forming by stretch & 144 & 0.198 \\
\hline B: forming by shear, single step method & 132 & 0.187 \\
\hline C: forming by shear, incremental method & 131 & 0.184 \\
\hline
\end{tabular}

It is now tempting to draw conclusions from these results about what is the best method. However that is not justified. We cannot use this technique and draw conclusions from what is in our eyes the best fit. Only direct physical evidence can decide to what level shear is occurring in the 
actual forming operation, and from that what is the correct method to establish the equivalent strain. This example is only presented here to emphasize the differences that may result from the selected method.

The relevancy of these differences depends very much on the actual situation and our aim. If the strains are only used to get an idea of the level of deformation it is not important, however if the strains are used for more fundamental study of the material behaviour - which forms the basis for this paper - it is essential to distinguish the different definitions of 'major strain'.

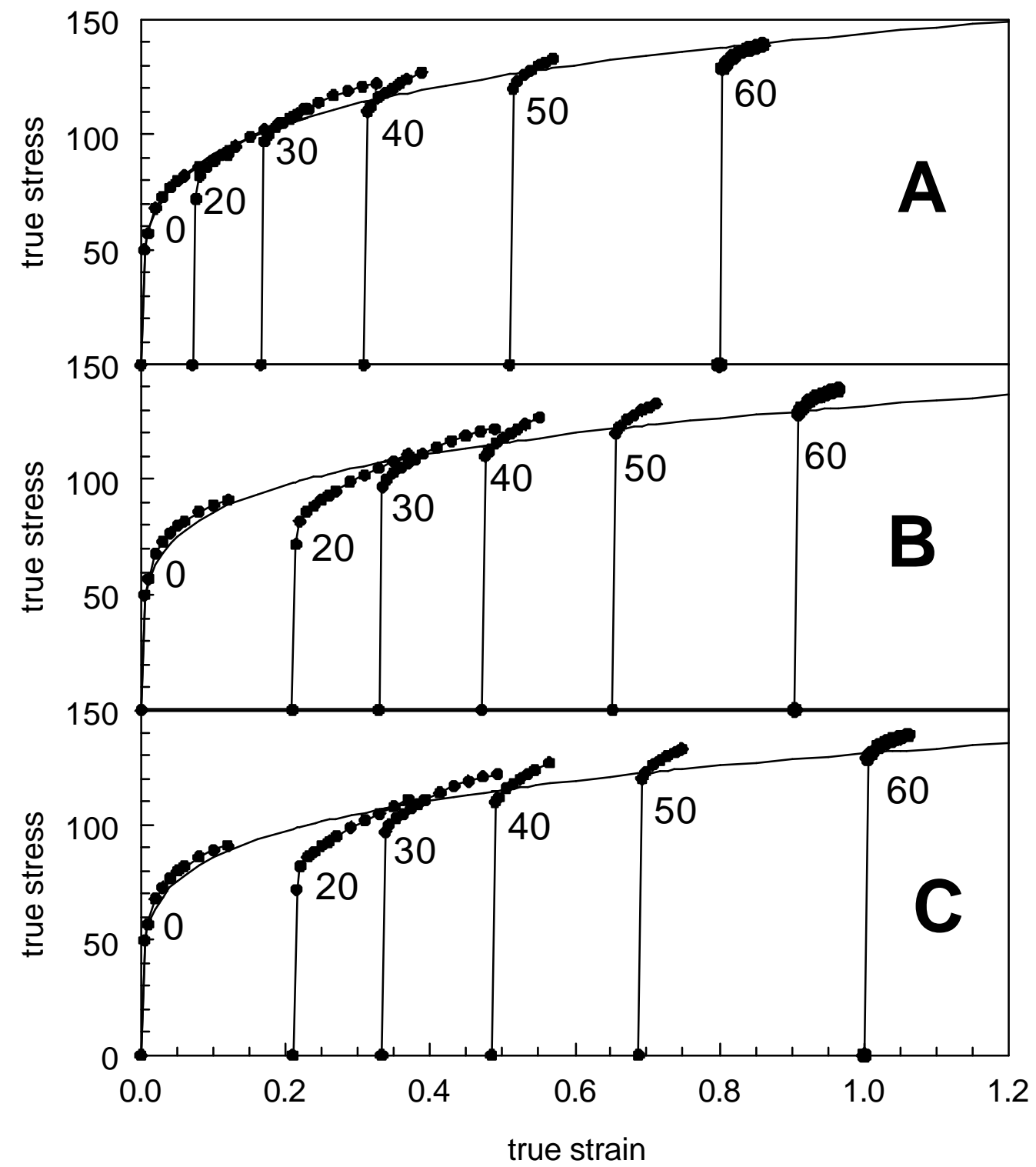

Figure 9. Tensile test data from pre-strained material [5] plotted with different values for the offset. The bbels 0, 20, 30, 40, 50, 60 denote the shear angle as defined in Fig. 2, 0 meaning undeformed material. The thin line represents a Hollomon fit through the data, the parameters of the fit can be found in Table 1.

A (top): offset strain according to forming by stretch

B (centre): offset strain according to forming by shear, single step method

C (bottom): offset strain according to forming by shear, incremental method.

\section{Discussion}

The calculations presented above yielded two different values for the strain at shear, and that is confusing. Remind that the strain was determined as a measure that controls (for example) the level of 
work-hardening in the material. The incremental method is mathematically correct, but that does not imply that it provides also a correct description of the material behaviour. The behaviour of a material under conditions of a constantly changing strain path is extremely complex and little is known about that (the only exception is the Bauschinger effect that has been studied in detail). It is only save to say that method B supplies a lower limit. Much more research is needed in this field.

Quite another question is if forming by shear will occur or not. Only direct physical evidence can answer that question. However we may assume that in every practical situation where there is bending some amount of shearing will take place, whether large or small. The behaviour of material under shearing is also little understood. This does not concern the behaviour on a micro scale but the technological, macroscopic behaviour. The behaviour of sheet under (more or less) flat stretching has been studied well, and we know that the process limits are determined by the onset of necking. The latter is caused by the simple fact that the material is being pulled. If we do not pull but push necking will not occur and the technological behaviour will be principally different. This implies that possibly forming by shear, which is neither pulling nor pushing, also may cause a different macroscopic behaviour in which necking does not occur or at least at other levels than in flat stretching. More research on the subject of forming by shear is required.

A problem may also arise when performing and verifying FEM calculations. A common verification technique is to compare predicted von-Mises strain to measured values. It might be assumed that the FEM code calculates the von-Mises strains correctly from the complex 3D-strain path. If there is indeed some amount of shearing present then the measured values obtained by simple grids on the sheet surface will be different (generally: lower). This can give an unjust impression of inaccuracy.

The main conclusion is that, now that a whole family of forming processes has become available that are supposed (or assumed) to act under forming by shear, we have to empty our minds and start thinking from scratch again.

\section{References}

[1] J. Jeswiet. Asymmetric Incremental Sheet Forming. Proceedings 11th Shemet, Apr 05-08, 2005, Erlangen, Germany, pp 35-58

[2] T.J. Kim, D.Y. Yang. Improvement of formability for the incremental sheet metal forming process. Int. Journal of Mechanical Sciences 42 (2000) 1271-1286

[3] L.E. Malvern; Introduction to the mechanics of a continuous medium; Prentice-Hall Inc. Englewood Cliffs, New Jersey, 1969

[4] A.H. van den Boogaard; Thermally Enhanced Forming of Aluminium Sheet; Thesis University of Twente, the Netherlands, 2002

[5] E. Hagan, J. Jeswiet. Effect of wall angle on Al 3003 strain hardening for parts formed by computer numerical control incremental forming. Proc. Inst. Mech. Engnrs vol 217(2003), Part B: J. Engineering Manufacture, pp 1571-1579 